\section{Opportunities and Considerations for Pharmacist Intervention in the Management of the Chronic Hepatitis C Patient}

Hepatitis $\mathrm{C}$ virus (HCV) is a major cause of morbidity and mortality; it may lead to chronic hepatitis, cirrhosis, hepatocellular carcinoma (HCC) and is a leading indication for liver transplantation. ${ }^{1} \mathrm{HCV}$ affects 300 million people worldwide and an estimated 3.9 million Americans have been exposed to 1 of the 6 genotypes of the HCV. ${ }^{1}$ Approximately $75 \%-85 \%$ of patients infected with hepatitis $C$ will develop a chronic HCV infection. ${ }^{1}$ Unfortunately, $10 \%-20 \%$ of chronically infected HCV patients will have their disease progress to cirrhosis and are at increased risk for decompensated liver disease. ${ }^{1}$ The staggering number of those exposed and consequently progressing to chronic HCV infection illustrates the importance of effective treatment and support. As in many other chronic illnesses with detrimental sequelae (e.g., coronary artery disease, diabetes and hypertension), pharmacists may be a resource to manage the disease and mitigate negative outcomes. ${ }^{2}$

In the March 2009 issue of JMCP, Mariño et al. presented the results of their assessment of a pharmacist intervention in treatment of genotype 1 chronic HCV patients. ${ }^{3}$ They described a multidisciplinary team in which pharmacists provided drug therapy management; education, including information about storage, methods for drug administration, management for potential drug reactions and missed doses; and support. ${ }^{3}$ In the same issue, Spooner discussed the potential benefits of, and highlighted barriers to, pharmacist involvement in HCV management. ${ }^{4}$ Spooner reinforced the perception that pharmacists could naturally fill the role of providing care for patients with HCV in a way that is similar to their already established roles in management of other chronic disease states. ${ }^{4}$

\section{Ethnicity and Patient-Specific Considerations}

The article by Mariño et al. focused on the most prevalent HCV genotype, genotype 1 . The overall rate of sustained viral response (SVR) rate demonstrated by Mariño et al. was 48\%, which is consistent with previously reported studies in genotype 1 patients. ${ }^{3,5,6}$ The authors surmised that the SVR rate could be partially attributed to the pharmacist interventions intended to increase adherence rates. ${ }^{3}$ Mariño et al. shed further light on the importance of the pharmacist's role on the multidisciplinary team in the care of the difficult-to-treat genotype $1 \mathrm{HCV}$ patient. ${ }^{3}$ However, the Mariño et al. study population captures, via convenience sample, a homogenous patient population that may not be representative of the genotype 1 patients treated in the United States. ${ }^{3}$ In addition to genotype, it is essential to consider other population characteristics when managing HCV treatment and assessing outcomes of interventions. For example, risk factors for heart disease, hypertension and diabetes mellitus continue to increase among African Americans, creating a patient population with multiple comorbidities. This diversity, within the genotype 1 chronic hepatitis $C$ population, was not captured in the patient population studied by Mariño et al. and their findings therefore may not be valid for patient populations in the United States. In contrast to the Mariño et al. study population, the U.S. HCV population includes a growing number of African Americans with HCV, HIV coinfected individuals, as well as patients with multiple other chronic conditions such as diabetes, cardiovascular disease, and hypertension. ${ }^{7,8}$ In addition to the size and the heterogeneity of the population, genotype 1 chronic HCV infection is often compounded by history of previous alcohol or drug abuse and the potential for adverse reactions or drug interactions while on treatment as well as the comorbidities mentioned above. ${ }^{8}$

The prevalence of the disease differs across various ethnic groups, with genotype 1 being the most common genotype among the African American population,, and the prevalence of $\mathrm{HCV}$ infection is about 3 times higher in black individuals than in white individuals. ${ }^{78}$ In addition, a case-control study suggested that the risk for liver cancer doubled among African American patients compared with the white cohort. ${ }^{9}$ The increased risk of disease progression to end-stage liver disease (cirrhosis) or the need for transplant should mean that treating the African American patient infected with HCV is associated with a greater potential cost savings. Unfortunately, several retrospective studies have indicated that African Americans have lower SVR rates than whites. ${ }^{7,8}$ Some researchers postulate that differences in immune response may be responsible for the disparity in SVR. ${ }^{10}$ Therefore patients and health care providers should be more motivated to achieve viral eradication in this patient population. In a setting that utilizes a pharmacist as a part of the clinical health care team, the role of the pharmacist as a health care provider should be geared toward educating patients not only on the treatment options but also on the benefits of treatment as it relates to prevention of disease progression.

\section{Protocol-Based Collaborative Practice}

Our ambulatory care chronic HCV clinic is a protocol-based collaborative practice. This practice includes a dedicated pharmacist working in partnership with physicians to manage all patients receiving treatment for chronic $\mathrm{HCV}$ infection. The clinical pharmacist is responsible for baseline assessment for contraindications to therapy, injection training, documenting receipt of recommended Hepatitis A and B immunizations, and ordering, based on protocol and with a physician's cosignature, medications and the laboratory tests necessary for monitoring toxicity and efficacy of therapy. Patients have access to a dedicated hotline available 24 hours per day, where they may talk to a clinical pharmacist or leave a confidential message and receive a response within 24 to 48 hours. In addition to office visits, patient can also complete telephone appointments and have access to their physician and clinical pharmacist via a secure Internet process. Within the clinic setting, the clinical pharmacist also precepts pharmacy interns, students, and residents. The clinical pharmacist manages the drug therapy for all chronic HCV patients, including those with comorbid conditions (e.g., congestive heart failure, 
cardiovascular disease, diabetes mellitus, hypertension) and those who are coinfected with human immunodeficiency virus (HIV).

Pharmacists in this collaborative practice also play an important role as vaccine advocates. The immunization status of the enrolled population was not addressed by the researchers in the report by Mariño et al. ${ }^{3}$ The Centers for Disease Control (CDC) Advisory Committee on Immunization Practices adult immunization schedule recommends influenza, pneumococcal, and hepatitis A and B vaccination for individuals with chronic liver disease who lack immunity. ${ }^{11}$ In their discussion of the implications of chronic HCV for managed care, McHutchison et al. reiterated that vaccination is "considered to be the most important tool in the prevention of chronic viral infections," and suggested that immunizations with hepatitis A and hepatitis B vaccines should be offered. ${ }^{12}$

The mutual risk factors (intravenous drug use, multiple sexual partners) for HCV and HIV have resulted in a unique but growing population of HIV patients living longer with the help of highly active anti-retroviral therapy (HAART) who are now being forced to deal with the long-term effects of HCV coinfection. HIV coinfected patients were excluded from the Mariño et al. study population. ${ }^{3}$ Spooner appropriately assessed that this exclusion may have eliminated from the Mariño et al. sample a large portion of the HCV-infected population. ${ }^{4}$ Pharmacist interventions in the HIV/HCV coinfected patient population, as part of the multidisciplinary team, offer balance in managing high-risk drug therapies in which adherence is critical to achievement of desired outcomes. In the assessment of HIV/HCV coinfected patients, Bräu documented that those patients with ongoing HIV viremia have a faster rate of HCV-related progression to liver fibrosis and liver failure than do HCV monoinfected patients. ${ }^{13}$ Health care providers must take into consideration the fact that HIV may accelerate the disease progression of HCV and that a substantial number of medications used to treat HIV may be hepatotoxic. The value of a pharmacist in the management of the coinfected patient increases exponentially because of the often complex drug therapy regimens and increased potential for adverse drug reactions, drug-drug and drug-disease interactions. In addition to participating in the management of the disease process as part of a multidisciplinary team, the pharmacist can further contribute by assessing cost-effectiveness, as appropriate.

In 2007, McHutchinson et al. projected an increasing number of individuals with chronic $\mathrm{HCV}$, detailing the potential health and economic burdens associated with the expected doubling of morbidity and mortality rates related to HCV liver sequelae over the next 20 years. ${ }^{12}$ In 2000, Wong et al's economic model projected $\$ 10.7$ billion in direct medical costs over a 9-year period from 2010 to $2019 . .^{14}$ In a commentary regarding the managed care considerations of chronic HCV, McHutchison et al. observed that when there is ongoing support by a clinical team it has been demonstrated that patients are more likely to adhere and complete therapy. ${ }^{12}$

We have noted in our hepatitis $C$ clinic that some patients are opting to defer drug therapy until they feel more financially secure. The current economic climate creates apprehension as patients worry that missed days or under performance at work may result in loss of income and possibly health insurance benefits, not just for themselves but also for their families. As is the case in other chronic disease states patients must consider the costs of the treatment including indirect cost like missed days from work, the decrease in overall quality of life, and potential cost of therapy to treat adverse reactions. These considerations are exacerbated by the limited potential for a return on this investment as there is approximately a $40 \%-50 \%$ probability of SVR in genotype 1 with combination drug therapy in controlled trials with ribavirin and pegylated interferon, depending on compliance and the specific regimen used. ${ }^{6}$

The adverse drug reaction profile for pegylated interferon and ribavirin therapy is extensive; patients being treated for genotype 2 and 3 disease may have to endure these reactions for only 24 weeks, but patients treated for genotype 1 disease will need treatment for at least 48 weeks. ${ }^{15}$ In addition to the multiple potential adverse drug reactions, the treatment of chronic HCV infection is time consuming, labor-intensive for health care providers and expensive. A multidisciplinary approach to the treatment of the $\mathrm{HCV}$ infected patient facilitates the availability of a member of the health care team to adequately manage side effects, drug interactions, and the heightened need for therapeutic monitoring in this patient population. In her commentary, Spooner highlighted the monetary considerations and lack of awareness of opportunities that sometimes prevent the use of a clinical pharmacist in HCV management. ${ }^{4}$ However, as described by Mariño et al., pharmacists are in a unique position to identify and respond to potential opportunities for improvement in medication use such as side effect recognition and management, as well as providing education regarding appropriate dose administration. ${ }^{3}$ As the recognized drug expert, the pharmacist is uniquely qualified to conduct therapeutic and adverse reaction monitoring (e.g., viral load and liver function test changes), as well as watching for drugdrug, and-more often overlooked-drug-disease interactions and provide therapeutic alternatives at patient-specific doses.

The experience in our clinic suggests that the pharmacist can also complete portions of the baseline assessment, facilitate patient education classes and be another resource that is accessible to the patient for support throughout the course of a difficult therapy regimen. The patients in our clinic who develop significant anemia due to the hemolytic effect of ribavirin are often offered epoetin alfa to restore the balance between red blood cell production and destruction without discontinuing or reducing the dose of ribavirin; once again affording the patient the best opportunity for SVR. Epoetin alpha is an injectable medication that requires extensive one-on-one counseling in order to afford patients the autonomy to effectively self-administer this therapy at home. The clinical pharmacist in our practice setting provides vital patient-specific drug and disease education, specifically proper injection technique and signs of potential adverse 
reactions. This education is vital because the use of epoetin alpha is not without risks; its use has been linked with significant adverse reactions like potentially fatal blood clots. ${ }^{16}$ For this reason it is important that patients receiving this medication be educated about signs and symptoms of clots and have their hemoglobin monitored closely to reduce these risks, in our practice as often as every other week.

Pharmacists are in the opportune position to motivate patients, stress adherence, and work with providers to reduce and or manage side effects to optimize treatment responses, especially in a genotype 1 patient. In our setting the pharmacist completes patient encounters geared towards disease state education prior to initiation of chronic HCV drug therapy. That education focuses on sequelae of chronic HCV infection including cirrhosis, HCC, decompensated liver disease, the need for transplant and possibly death. During these and future patient encounters the pharmacist also reviews drug therapy, adverse reactions, duration of therapy, incidence of SVR and treatment response markers. Following initial encounters patients are encouraged to take the opportunity to think about their treatment options and discuss with loved ones. Throughout the course of therapy patients have access to both their physician and pharmacist for general questions and to assist in managing adverse reactions. An integral part of the pharmacist function also includes proactive interview of patients regarding side effects, ongoing review of medication dispensing history and encouragement of pillbox utilization. In addition, at all encounters patients are encouraged lean on their multidisciplinary team as part of their treatment support system.

One of the main challenges for the future is to improve success rates for difficult-to-treat HCV genotype 1 patients. ${ }^{5}$ At this time, there is no vaccine for $\mathrm{HCV}$, so spread is controlled only by universal infection precautions. Since disease progression can be severe and costly (directly and indirectly) to manage, effective disease management strategies are important. In our specialized hepatitis clinic that involves a physician-pharmacist partnership, a major portion of the care of patients with chronic hepatitis $\mathrm{C}$ revolves around medication adherence and adverse effect management. Further studies are needed to quantify the relationship between pharmacist interventions and improvements in SVR, adherence and cost-effectiveness. Data that quantify the cost of treatment, or lack of treatment, of chronic HCV infection in high risk (i.e., African American, HIV coinfected) patients would also be valuable as this patient population continues to grow.

\section{Nafesa Walters-Smith, PharmD, BCPS \\ Clinical Pharmacy Assistant Manager \\ Kaiser Permanente of Georgia, Atlanta, Georgia \\ Nafesa.Walters-Smith@kp.org}

\section{S. Renee Marshall, PharmD}

Infectious Diseases Clinical Pharmacy Specialist Kaiser Permanente of Georgia, Atlanta, Georgia Sherry-Ann.R.Marshall@kp.org

\section{DISCLOSURES}

The authors disclose no bias or conflict of interest related to the subject of this commentary.

\section{REFERENCES}

1. Center for Disease Control and Prevention (CDC). Recommendations for prevention and control of hepatitis C virus (HCV) infection and hcv-related chronic disease. MMWR Recomm Rep. 1998;47(RR-19):1-39.

2. Pellegrino AN, Martin MT, Tilton JJ, Touchette DR. Medication therapy management services: definitions and outcomes. Drugs. 2009;69(4):393-406.

3. Mariño EL, Alvarez-Rubio L, Miró S, et al. Pharmacist intervention in treatment of patients with genotype 1 chronic hepatitis C. J Manag Care Pharm. 2009;15(2):147-50. Available at: http://www.amcp.org/data/jmcp/147150.pdf.

4. Spooner, L. The critical need for pharmacist involvement in the management of patients with hepatitis C. J Manag Care Pharm. 2009;15(2):151-53. Available at: http://www.amcp.org/data/jmcp/151-153.pdf.

5. Manns MP, Wedemeyer H, Cornberg M. Treating viral hepatitis C: efficacy, side effects and complications. Gut. 2006;55(9):1350-59.

6. Manns MP, McHutchison JG, Gordon SC, et al. Peginterferon alfa$2 \mathrm{~b}$ plus ribavirin compared with interferon alfa- $2 \mathrm{~b}$ plus ribavirin for initial treatment of chronic hepatitis C: a randomised trial. Lancet. 2001;358(9286):958-65.

7. Pyrsopoulos N, Jeffers L. Hepatitis C in African Americans. J Clin Gastroenterol. 2007;41(2):185-93

8. Bacon BR, McHutchison JG. Treatment issues with chronic hepatitis C: special populations and pharmacy strategies. Am J Manag Care. 2005;11(10 Suppl):S296-S306.

9. Nguyen MH, Whittemore AS, Garcia RT, et al. Role of ethnicity in risk for hepatocellular carcinoma in patients with chronic hepatitis $C$ and cirrhosis. Clin Gastroenterol Hepatol. 2004;2:820-24.

10. Muir AJ, Bornstein JD, Killenberg PG, et al. Peginterferon alfa 2b and ribavirin for the treatment of chronic hepatitis $\mathrm{C}$ in blacks and non-Hispanic whites. N Engl J Med. 2004;350: 2265-71.

11. Centers for Disease Control and Prevention Advisory Committee on Immunization Practices Recommended Adult Immunization Schedule, United States - 2009. Available at: http://www.cdc.gov/vaccines/recs/schedules/adult-schedule.htm. Accessed March 30, 2009.

12. McHutchison JG, Bacon BR, Owens GS. Making it happen: managed care considerations in vanquishing hepatitis C. Am J Manag Care. 2007;13(12 Suppl):S327-S336.

13. Bräu N. Treatment of chronic hepatitis $C$ in human immunodeficiency virus/hepatitis $C$ virus-coinfected patients in the era of pegylated interferon and ribavirin. Semin Liver Dis. 2005;25(1):33-51.

14. Wong JB, McQuillan GM, McHutchison JG, Poynard T. Estimating future hepatitis C morbidity, mortality, and costs in the United States. Am J Public Health. 2000;90:1562-69.

15. Strader DB, Wright T, Thomas DL, Seeff LB; American Association for the Study of Liver Diseases. Diagnosis, management and treatment of hepatitis C. Hepatology 2004;39(4):1147-71.

16. Procrit [package insert]. Thousand Oaks, CA: Amgen Inc. August 2008. Available at: http://www.procrit.com/procrit/shared/OBI/PI/ProcritBooklet. pdf. Accessed April 27, 2009. 